\title{
AN ADDITIONAL STIFFNESS PARAMETER MEASURE OF ERROR OF THE SECOND KIND IN THE FINITE ELEMENT METHOD
}

\author{
GANGAN PRATHAP ${ }^{\dagger}$ \\ Structural Sciences Division, National Aeronautical Laboratory, Bangalore, India
}

\section{SUMMARY}

Conventional error analyses norms in the finite element method are based on the percentage error or its equivalent in some computed value as compared to the theoretically predicted value. In problems where two or more field variables are coupled, it is possible that if the field interpolation functions do not 'consistently' reproduce limiting situations of physical behaviour, there may be very large errors which are exaggerated enormously when some structural parameters become indefinitely large in a penalty limiting sense. The percentage error norms therefore saturate quickly to a value approaching 100 per cent and do not sensibly reflect the relationship between error and the structural parameter, even on a logarithmic plot.

Errors due to these spurious constraints have recently been recognized as belonging to a special class described as 'errors of the second kind'. A new error norm, called the additional stiffening parameter, helps to recognize the manner in which errors of the second kind canbe blown out of proportion by a large variation in some structural parameter. Recent investigations in some multi-field problems are summarized here to demonstrate the usefulness of this concept.

\section{INTRODUCTION}

Over the past decade, it has become clear that in many finite element formulations of continuum problems which are described by more than one field variable, severe constraints may be imposed artificially in certain limits as a result of the various mathematical operations involved in the discretization process, i.e. the division into small but non-infinitesimal subdomains, the definition of shape functions as approximations for the field variables over these regions and the integration of the strain-energy functional over these regions.

Such problems have been well documented as 'parasitic shear' in the plane stress modelling of beam flexure by two-dimensional plane stress and three-dimensional brick elements, ${ }^{1,2}$ 'shear locking' in shear-flexible beam, plate and shell elements, ${ }^{3-9}$ 'membrane locking' in arch/shell elements, ${ }^{10,11}$ etc.

Many means of alleviating these severe constraints have been explored, e.g. reduced integration, ${ }^{1,3-6}$ use of mixed and hybrid formulations, ${ }^{2,7,8}$ etc. Often, it is not recognized that some spurious form of constraint gives rise to special errors which we shall define in the next section as 'errors of the second kind' and that some technique such as described above is required to remove this error.

${ }^{\dagger}$ Presently at: Institut fur Strukturmechanik, DFVLR, 3300 Braunschweig, W. Germany. 
In this paper, we develop a simple basis for classifying 'errors of the second kind' which arise due to the "spurious constraints" imposed by certain formulations. We show how simple error models basal on this error estimate may be related to the geometrical/structural parameters that cause a severe deterioration of results when these parameters take large values in a limiting penalty function sense.

\section{ERRORS OF FIRST AND SECOND KIND}

It is now necessary to distinguish the special errors that arise due to the 'spurious constraints' from the other, more familiarerrors of discretization. We recognize that in a continuum problem with only a single field variable, the errors of discretization are of simple form and these usually vanish rapidly as the mesh size is reduced. However, in a multi-field problem in which, at limiting cases, constrained strainfields must be enforced, the 'spurious constraints' can give rise to a form of errors that vanish very slowly with reduction in mesh size, and whose slowness of convergence and magnitude of error are greatly exaggerated by changes in the structural parameters that emphasize the appearance of the limiting physical situations.

It will be appropriate to introduce a terminology that can recognize the existence of these two kinds of errors. Walz et al., ${ }^{12}$ in their classical order of error analysis based on the finite difference equivalents of finite element formulations of field problems, recognized the existence of twotypes of errors which they classified as follows-a first kind which vanishes as the element sizes are diminished and a second kind where the errors do not vanish with vanishing element size. Prathap and Bhashyam, ${ }^{9}$ in their work on the shear deformable beam element using continuum equivalent expressions of discretized functionals for the strain energy in a beam element, showed that both errors vanished with diminishing element size, but differed in that errors of the first kind were virtually independent of structural parameters used in the mesh idealizations, whereas the errors of the second kind could be exaggerated to large values when the structural parameters took large values in a penalty limit sense. Based on this interpretation, it was possible to derive error models which could accurately predict the deterioration caused by the severe constraints arising from the exactly integrated shear flexible (Timoshenko) beam elements.

Recently, these concepts were extended to cover the 'in-plane' or 'membrane locking' behaviour. of thin curved beams ${ }^{10}$ and the 'parasitic shear' induced in plane stress models of beam flexure. ${ }^{13}$ In the sections that follow, we unify some of the recent advances in this area and show how a simple additional stiffness parameter concept can be used to recognize the influence of the errors of the second kind, where they are present.

\section{THE SHEAR DEFORMABLE BEAM ELEMENT}

Although the effectiveness of reduced integration in finite element applications was first recognized in the two-dimensional plane stress modelling of beam flexure ${ }^{1}$ and in subsequent applications to shear flexible plates and shells, ${ }^{3,4}$ it was only after the simplest shear-deformable beam bending element was formulated and tested ${ }^{5}$ that a heuristic argument for the success of reduced integration was advanced. An independently interpolated displacement model of a shear flexible beam element with 2 nodes and 2 degrees-of-freedom at each node, the transverse displacement $w$ and the face rotation 9, would have linear interpolation functions of the type

$$
\begin{aligned}
& w=a_{1}+a_{2} x \\
& \theta=b_{1}+b_{2} x
\end{aligned}
$$


The evaluation of shear strain energy is now based on the expression for shear strain:

$$
\begin{aligned}
\gamma & ={ }^{n}-w_{, x} \\
& =\left(u_{2}+b_{1}\right)+a_{2} x
\end{aligned}
$$

Both Hughes et al. ${ }^{5}$ and Lee and Pian ${ }^{7}$ recognized that in the extreme penalty limit of a thin beam modelled by elements of length $L$ and thickness $t$, two constraints are imposed, namely

$$
\begin{array}{r}
a_{2}+b_{1} \\
a_{2}=0
\end{array}
$$

The early heuristic arguments advanced related to the fact that these two constraints were present among 4 degrees-of-freedom and that these were too many and caused the locking of the solution in the very thin limits. However, it was not clear then that if just one constraint was correct among 4 degrees-of-freedom per element, how this constraint was to be chosen, or how reduced integration will select the correct constraint and reject the spurious constraint.

Prathap and Bhashyam ${ }^{9}$ separated the constraints into two classes: a true constraint which correctly reproduces the Kirch hoffconstraint with terms from both interpolation functions, i.e. the first constraint in equation (3) above, and a spurious constraint in which only a term from one interpolation function (in this case, the face rotation 9) tended to enforce a spurious restriction on the behaviour of that field in the extreme limits of thin beam behaviour, i.e. the second constraint in equation (3) above. In this context, they were able to derive models of the constrained behaviour in the thin beam regime, when exact numerical integration of the shear energy is adopted. These models could predict the exact quantitative nature of the errors of the second kind.

In this section, these error models are reinterpreted on the basis of a new measure called the additional stiffness parameter $e$. Crucial to this definition is the observation that the exact integration of the shear strain energy results in an 'inconsistent' representation of the shear strain field. The 'inconsistent' part is the linear contribution $a_{2} x$ which emerges only from the interpolation function of one field variable. This contributes a spurious bending energy of the form $k G A L^{2} \theta_{, x}^{2}$. In effect, this alters the original bending rigidity / to a value /' given by

$$
I^{\prime}=I\left(1+k G L^{2} / E t^{2}\right)
$$

for a beam element of rectangular cross-section. The $k G L^{2} / E t^{2}$ term is now the structural parameter that enforces the spurious constraint $a=0$ in the penalty limit of extreme thinness (i.e. $L / t \rightarrow \infty$ ). Alternatively, this can be thought of as a spurious stiffening of the beam rigidity in the manner expressed by equation (4). This suggests that the quantity

$$
\left(I^{\prime}-I\right) / I \simeq k G L^{2} / E t^{2}
$$

would be the structural parameter that could be used to measure the degree of 'shearlocking' in the model.

Thus, a new error parameter which will reflect errors of the second kind can be defined in the form

$$
e=\frac{w \text { (theory) }}{w(\text { fem })}-1
$$

where $\mathrm{w}$ is a reference deflection under some static loading condition. In the case ofa shear flexible cantilever beam of length / and thickness $t$ idealized by a fixed number of elements of equal length $L$, we can compare the tip deflection under a concentrated vertical tip load $P$ for the finite element model which has its shear strain energy exactly integrated, with the theoretically predicted value. Figure 1 re-plots a typical set of results for the beam element described in Reference 9, on a 


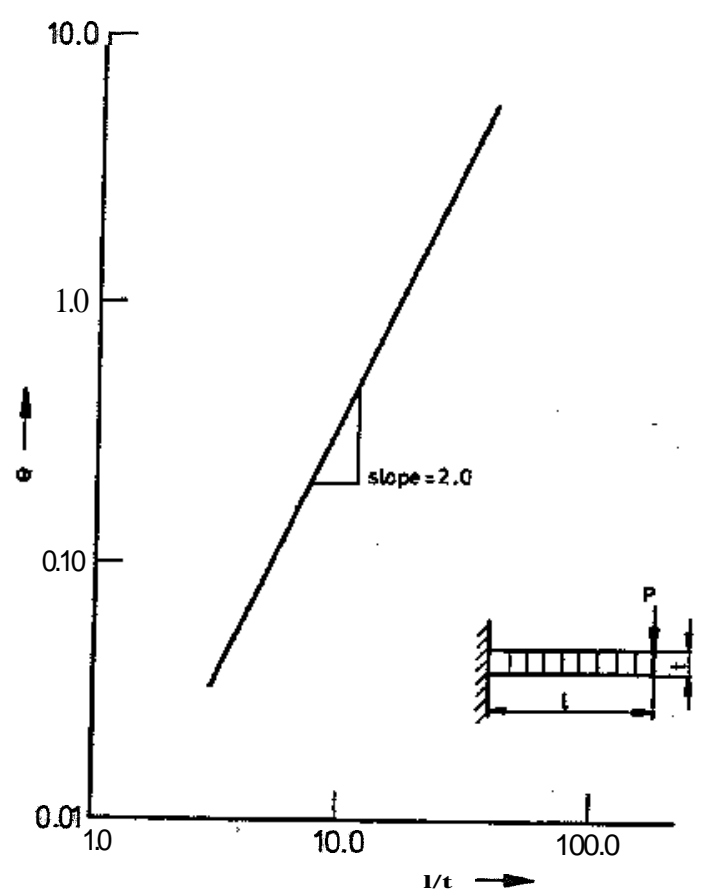

Figure 1. Additional stiffness parameter for a cantilever beam of length / and thickness $t$ idealized by $N$ elements

logarithmic plot. This plot relates $e$ to $l / t$. Since afixednumber of elements is used and the thickness $t$ is decreased to the limiting value, we expect the errors of the second kind to propagate in a $(L / t)^{2}$ and therefore $(l / t)^{2}$ fashion. Figure 1 shows that such a quadratic relationship is exactly predicted by the numerical results. In contrast, when the shear strain energy is evaluated using reduced integration (a one-point Gaussian integration is optimal in this case), the additional stiffness parameter is too small to be shown on this graph, is of the order of 0.003 and varies very little over the range of $l / t$ considered.

\section{EXTENSIONAL THIN ARCH/CURVED BEAM ELEMENTS}

Until very recently, the poor behaviour of arch/curved beam/finite circular ring elements was attributed to the inability of independent low order polynomial interpolations for the tangential and normal displacements to reproduce a strain free rigid-body motion. ${ }^{14,15}$ However, recent developments $^{9-11,16}$ show that it is the inability of these interpolations to 'consistently' model the membrane strain with terms from both interpolation functions that led to spurious 'in-plane' or 'membrane' constraints.

It $_{\text {is }}$ instructive, in this section, to re-examine a thin deeply curved beam so that the membrane or in-nlane locking action can be studied in isolation. A curved beam element with independent displacement fields which are linear in the tangential displacement $v$ and cubic in the normal displacement $w$ has been examined by several people and discarded because of its very poor behaviour in thin and deep situations. Lee and Pian ${ }^{7}$ observed that interpolations of the form

$$
\begin{aligned}
v & =a_{1}+a_{2} \phi \\
w & =b_{1}+b_{2} \phi+b_{3} \phi^{2}+b_{4} \phi^{3}
\end{aligned}
$$


would produce, in rigid-body motion behaviour, four constraints of the form

$$
\begin{aligned}
a_{2}+b_{1} & =0 \\
b_{2} & =0 \\
b_{3} & =0 \\
b_{4} & =0
\end{aligned}
$$

The heuristic argument brought in here is that among the 6 degrees-of-freedom available to each element, there are four constraints and that these are too many and would severely limit the performance of the element. The emphasis was again on the inability to represent rigid-body modes in a strain-free manner. These heuristic arguments remained inadequate in that they could not indicate how many of the constraints were enough and how many constituted an excessive number among 6 degrees-of-freedom. Also, there were no indications on how to choose the correct constraints.

A recent interpretation from Prathap ${ }^{10}$ showed that a treatment in terms of the need for a field consistent membrane strain representation could provide the correct clues. The first of the four constraints, equation (7a), was a true membrane constraint in that it had terms from both interpolation functions. The remaining three constraints, equations (7b)-(7d), are spurious ones. Consequently, it can be shown that a one-point. Gaussian integration of the membrane strain energy would correctly represent a true inextensional constraint in very thin limits and that any higher order of integration, i.e. two-point or above (a four-point rule would be equivalent to an exact integration in this case) would introduce spurious constraints that lock the solution.

A simple error model could be devised on this basis and predicts an error that varied quadratically with the $(\beta L / t)$ ratio, where $L$ is the length and $t$ the thickness of the element, and $\beta$, the angle

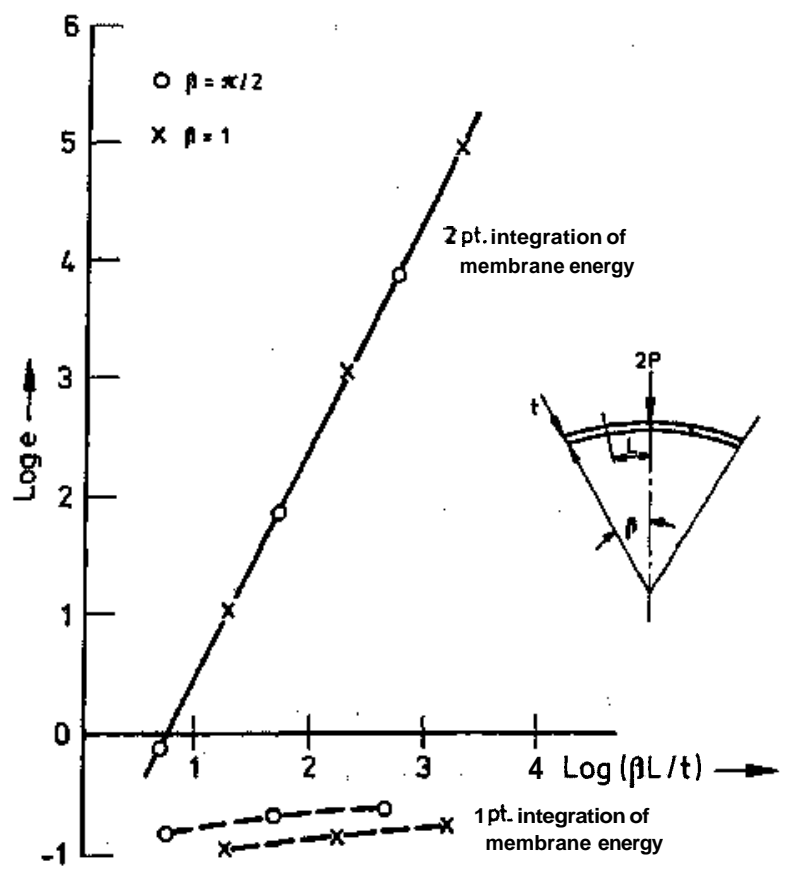

Figure 2. Additional stiffness parameter for clamped arch under central concentrated load 
subtended by the arch, a measure of the deepness or shallowness of the arch. ${ }^{10}$ Figure 2 shows the results for a clamped -clamped arch with a central concentrated load (see inset in Figure 2) and the error parameter $e$ is now based on the normal deflection $w$ under the load. Two arches of subtended angle $\beta=\mathrm{I} \mathrm{rad}$ and $\pi \mathrm{rad}$ were studied. With a one-point integration of the membrane energy, there was no rapid deterioration of results in the parameter $L / t$ over a range $3 \cdot 142$ to $314 \cdot 2$ and $20 \cdot 0$ to $2000-0$ in each case. However, with two-point and four-point integrations of the membrane energy, the locking is nearly identical (that is, too close to each other to be separated on the graph) and varies exactly in a $(\beta L / T) \hat{f}$ ashion, as predicted in Reference 10 . Clearly, any order of integration higher than the optimal one-point rule would bring in the spurious membrane constraints and these produce the in-plane locking errors of the second kind.

\section{SHEAR DEFORMABLE PLATE BENDING ELEMENTS}

Early work on the role of reduced integration in the 8-noded serendipity shell elements ${ }^{3,4}$ led to investigations into $C_{0}$ continuous Mindlin plate elements based on independent interpolation functions for the transverse deflectionw and the face rotations $\theta_{x}$ and $\theta_{y}$. It was found that reduced integration was imperative to generate a usable element in the moderately thick to the very thin regime. The first simple and successful plate bending element was that of Hughes et al., ${ }^{5}$ where a one-point Gaussian integration of the shear energy provided an efficient element. The early explanations for this success were offered in terms of the need for a singular shear stiffness matrix and/or in terms of the number of constraints introduced by the order of integration chosenwhether these were too excessive or not. However, an optimal integration strategy that would not

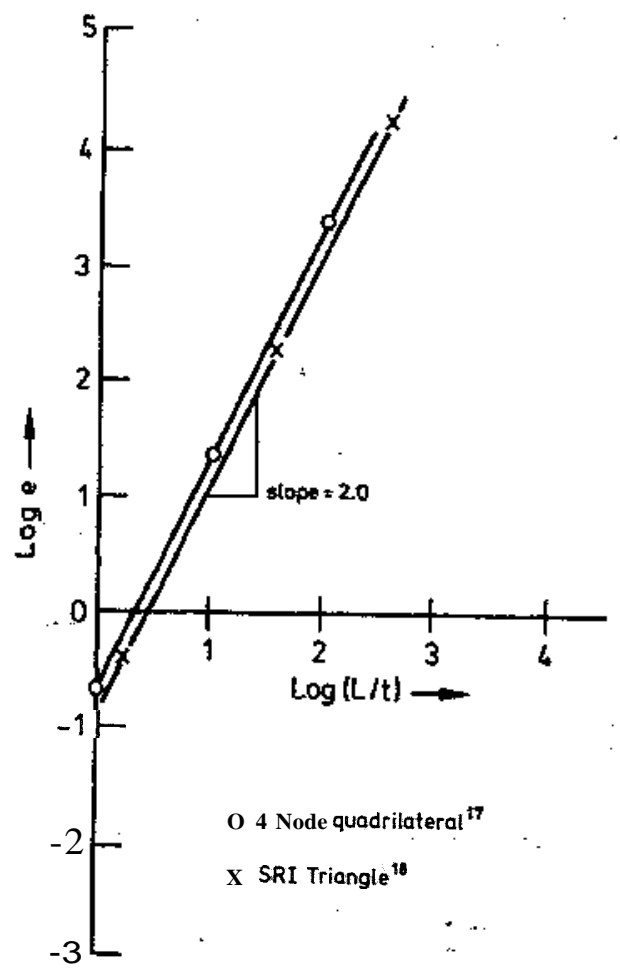

Figure 3. Additional stiffness parameter for square plate under central concentrated load 
suffer from zero energy mechanisms and that would retain all true Kirchhoffconstraints could be worked out only on the basis of a field consistency interpretation. ${ }^{17}$ This ensured that only those shear strain terms that were constituted of terms from both interpolation functions (i.e. for $w$ and $\theta_{x}$

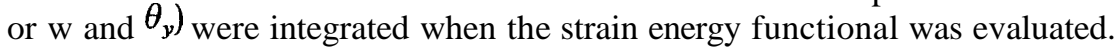

It is useful, now, to demonstrate that when exact integration of the shear strain energy is employed, the errors of the second kind propagate in a $(L / t)^{2}$ fashion, where $L$ is the element length used to idealize the mesh and $t$ is the thickness of the plate.

In Figure 3, we show the results obtained for a simply-supported square plate under the action of a central concentrated load. A quarter of the plate is idealized by a $4 * 4$ mesh of the bilinear plate bending element, ${ }^{5,17}$ with the shear energy evaluated by an exact numerical integration (i.e. a $2 * 2$ Gaussian integration rule). With decreasing thickness $t$, so that the very thin plate regime is covered in a limiting sense, the additional stiffness parameter is seen to explode in a $(L / t)^{2}$ fashion. Clearly, the spurious shear constraints emerging from the lack of field consistency when the shear strain energy is evaluated exactly tend to overemphasize a spurious shear strain energy which dominates the flexural energy in a $(L / t)^{2}$ fashion.

Also, in Figure 3, we reproduce for comparison results from a selective reduced integrated triangular plate bending element with transverse shear deformation. ${ }^{18}$ This is a $C_{0}$ continuous, 3noded, 9 degrees-of-freedom element based on independent linear interpolations for $w, \theta_{x}$ and $\theta_{y}$. It is presumed that a reduced integration of the shear energy would remove the spurious shear constraints and would produce an elegant, simple and effective plate bending element. However, it appears that a combination of two such triangular elements can generate a spurious shear constraint. ${ }^{19}$ This was recognized quite easily through the use of an additional stiffening parameter measure of error to demonstrate that an error of the second kind was indeed present. ${ }^{19}$ Figure 3 shows the result for a clamped square plate under a central concentrated load in which a quarter of the plate was idealized by a $12^{*} 12$ mesh. At such idealizations, no errors of the first kind can be expected. The additional stiffness parameter of the element, even after selective reduced integration was performed, did deteriorate in a $(L / t)^{2}$ fashion.

We see now that this factor is the basis of the residual energy balancing or penalty factor compensation techniques ${ }^{20-23}$ used often to de-emphasize errors of the second kind wherever they may be knowingly or unknowingly present. The word of caution to be introduced here is that, often, an incomplete application of reduced integration may not eliminate all spurious constraints.

We complete this section with a reassessment of the interesting results presented by Lee and $\operatorname{Pian}^{7}$ for an 8-noded isoparametric secrendipity plate bending element. We recognize that the R24 element which is based on a mixed Hellinger-Reissner formulation and the MR24 element which is based on a mixed modified Hellinger-Reissner formulation with a uniform $3 * 3$ integration are virtually identical to the conventional displacement type element with reduced integration $(2 * 2$ Gaussian integration) of the shear strain energy. Unexpectedly, the R24 and MR24 elements lock in a $(L / t)^{2}$ fashion in the thin plate region, as we see below. The MR24A element, which shows no locking, does not seem to have an optimal integration strategy for the 8-node displacement type quadrilateral.

Figure 4 gives the result for a simply-supported plate of side $a$ and thickness $t$ under a uniform pressure $p$ and with a $2 * 2$ mesh of R24 or MR24 elements idealizing a quarter of the plate. In the thin plate regime, where the spurious constraints are active, the error for both R24 and MR24 is seen to be identical and to vary exactly in a $(a / t)^{2}$ fashion. In the thick plate region, however, these terms cannot be interpreted as spurious constraints as they, in fact, constitute part of the true transverse shear energy. Consequently, in this regime, errors of the second kind do not play a critical role. Figure 5 shows a re-plot of the results in the thin plate regime on a logarithmic scale to demonstrate the $(a / t)^{2}$ nature of shear locking. The corresponding results for the MR24A element 


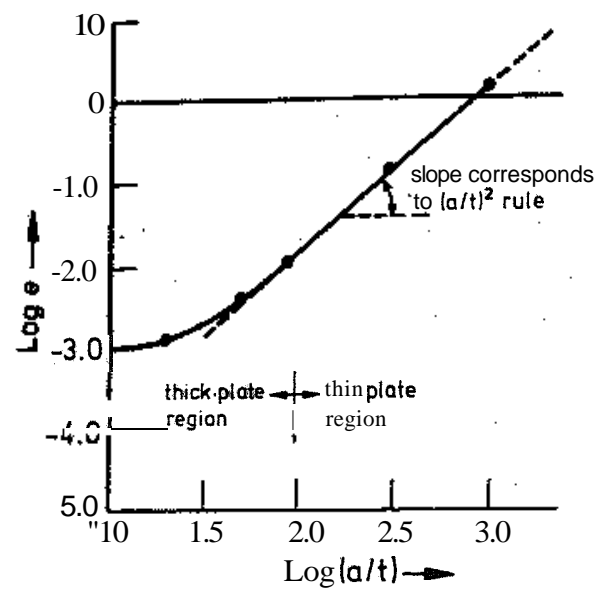

Figure 4. Additional stiffness parameter for square plate under uniformly distributed load (after Reference 7)

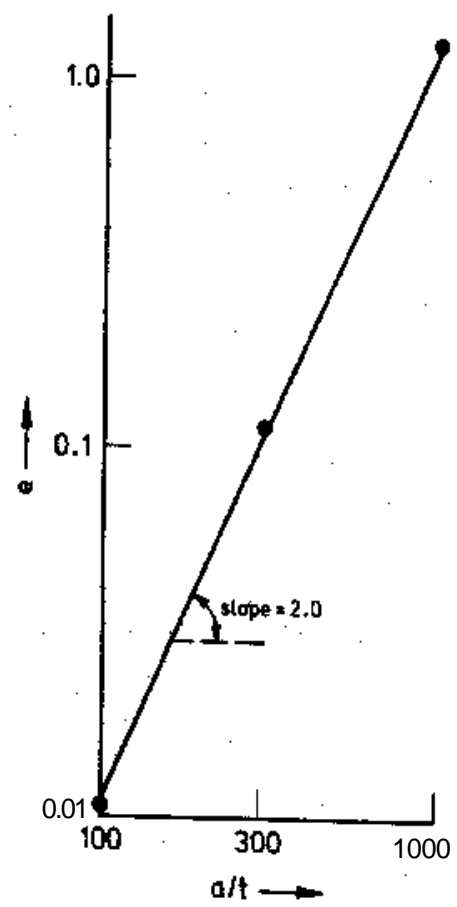

Figure 5 Additional stiffness parameter for square plate under uniformly distributed load in thin plate region (after Reference 7)

in Reference 7 shows that no error of the second kind is present and that the variation of the error parameter with $(a / t)$ is too small to be reliably portrayed in Figures 4 or 5 .

\section{PLANE STRESS MODELLING OF CANTILEVER BEAMS}

The very poor bending response of plane stress elements was te earliestrecognized instance of 'parasitic stear' or shear locking'. Early explanations for this were based on the inability of the 
quadrilateral shape of the linear 4-node element to bend into the simple curved shapes required for flexural modes. This provided a basis on which the remarkable improvement obtained by the addition of non-conforming modes was understood. ${ }^{24}$ It was also understood that, alternatively, a reduced integration of the shear strain energy of the original conforming quadrilateral element also produced an identical improvement.

It was only recently that the poor bending response of simple plane elements was considered in the context of field consistency theory. It was recognized that the independent interpolations for $u$ and $v$ could not model the shear stress field consistently in a flexural thin beam situation.

Figure 6 shows two idealizations of a beam of length / and depth $t$ idealized by rectangular plane stress elements of length $L$ and depth $T$. It was shown in Reference 13 that an exact integration of the shear strain energy in a flexural model would produce an inconsistent term which would lead to a spurious shear energy. This results in a locking of the form $G L^{2} / E t^{2}$. Curiously, this factor is independent of the number of elements used through the thickness, i.e. the element depth T, It is obvious that in limiting situations where $l / t$ can become very large, it is impossible to model the beam accurately with a small number of elements along the length of the beam.

It was also shown that where only one element was used through the thickness, a Poisson's effect emerged which altered the solution by a factor of $\left(1-v^{2}\right)$, a phenomenon well established in the literature. ${ }^{25}$ This, however, vanishes as the idealization through the thickness is increased, and does not constitute an error of the second kind in that it cannot be exaggerated by changing any structural parameter. The modified flexural rigidity of a beam shown in Figure 6, where only one
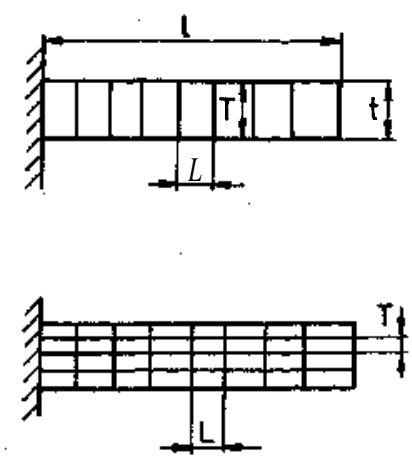

Figure 6. Plane stress models of beam undergoing flexure

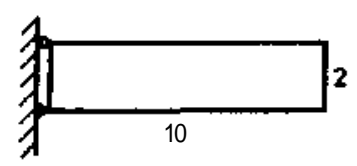

$$
\frac{d(\text { fem })}{d \text { (theory) }}=0.091
$$

\begin{tabular}{|c|c|}
\hline e(fem) & e (Eqn. 8) \\
\hline 10.0 & 10.1 \\
\hline
\end{tabular}
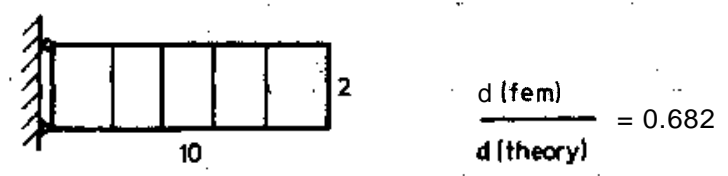

\begin{tabular}{|c|c|}
\hline e (fem) & e (Eqn.8) \\
\hline 0.47 & 0.47 \\
\hline
\end{tabular}

Figure 7. Additional stiffness parameter for an example from Reference 26 
ejerest is throngh the thickness, is of the form

$$
E I\left(1 /\left(1-v^{2}\right)+G L^{2} / E t^{2}\right)
$$

and, fora case

a large number of elements through the thickness has eliminated the Poisson's effect, $\mathrm{S}$

$$
E I\left(1+G L^{2} / E t\right)
$$

Therefore, the locking parameter is a function of the ratio $(L / t)$ and not $(L / T)$, the element aspect ratio. We demonstrate that ttts factor is indeed operative from typical results available in the literature.

Figure 7 shows a typical result for a beam analysed by the 4-node quadrilateral plane stress element. ${ }^{26}$ It was seen that equation (8) provides an excellent prediction for the $e$ values. Figure 8 shows a second example of a modeling by a 4-node quadrilateral, where again an error of the second kind is manifest and is predicted very accurately by equation (8). ${ }^{27}$

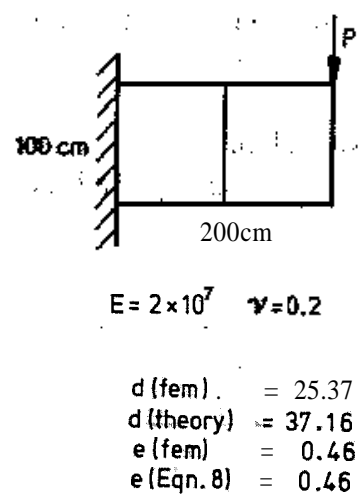

Figure 8. Plane stress model of beam undergoing flexure (Reference 27)

\begin{tabular}{|c|c|c|c|}
\hline \multirow[t]{2}{*}{$\therefore$} & wifeml & \multirow{2}{*}{ elfem] } & \multirow{2}{*}{ - Eqn. \& } \\
\hline & $w$ [theory] & & \\
\hline one ternent & 0.090 & 10.1 & 10.1 \\
\hline two ef thent & 0.278 & 2.6 & 2.6 \\
\hline
\end{tabular}

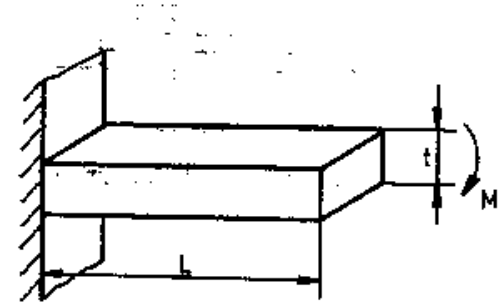

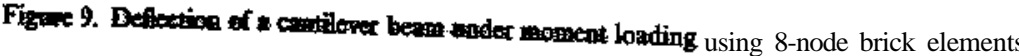




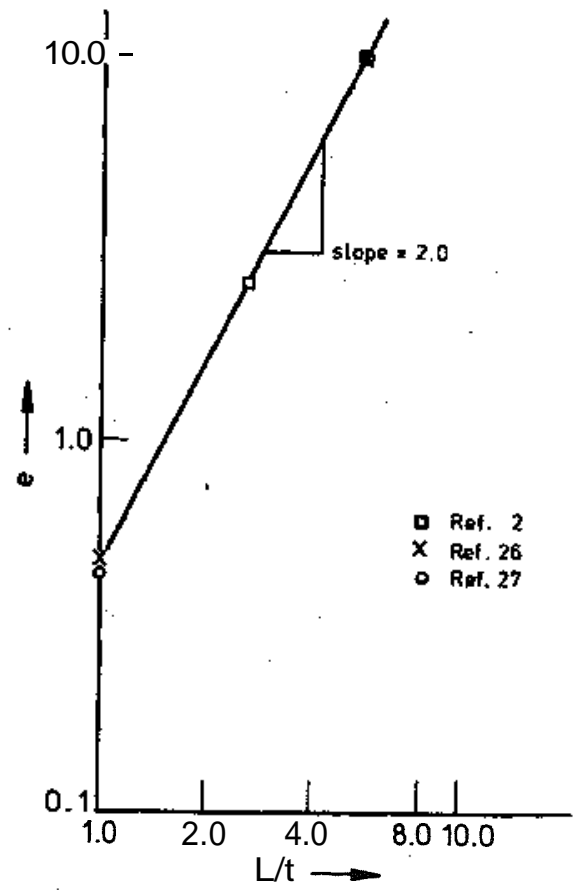

Figure 10. Additional stiffness parameter for the plane stress model of beam flexure

Figure 9 shows an interesting extension of equation (8) to predict the behaviour of a displacement-type 8 -node brick element with exact integration for the shear strain energy. ${ }^{2}$ Since the values of $v$ and $l / t$ are not indicated, it can be reworked, assuming that an error of the second kind as indicated by equation (8) is present. It is seen, in fact, that the one-element and two-element models show a behaviour consistent with equation (8), so that $G l^{2} / E t^{2}=10$ and $1 /\left(1-v^{2}\right)=1 \cdot 1$. With these, the additional stiffness parameters are as shown.

The three examples above are shown in a single plot in Figure 10 to demonstrate the $(L / t)^{2}$ factor in locking.

\section{CONCLUSIONS}

We have demonstrated, with examples, the use of an additional stiffening parameter to predict or detect errors of the second kind in problems where inconsistent field definitions can lead to very severe errors in certain limiting situations. Its application to several similar areas where overconstraining can result, e.g. incompressible fluid flow ${ }^{28}$ and incompressible elasticity ${ }^{29}$ will be interesting exercises.

\section{ACKNOWLEDGEMENTS}

The author is extremely grateful to Mr. B. R. Somashekar, Head, Structures Division, for his constant support and encouragement. The dedicated assistance of Mr. Ashok Kamath at all stages of the computational work is also gratefully acknowledged. 


\section{REFERENCES}

1. W P Doherty E L Wilson and R L Taylor, 'Stress analysis ofaxi-symmetric solids using higher order quadrilateral finite elements', Structural Engineering Laboratory Report No. SESM 69-3, University of California, Berkeley (1969).

2 T. H. H. Pian. D. P. Chen and David Kang, 'A new formulation of hybrid/mixed finite element', Comp.Struct., 16, 81-87 11983 .

3. O C. Zienkiewicz. J. Too and R. L. Taylor, 'Reduced integration technique in general analysis of plates and shells', Int.j. numer, methods eng.. 3, 275-290 (1971).

4. S. F. Pawsey and R. W. Clough, 'Improved numerical integration of thick shell finite elements', Int. j. numer. methods eng., 3, 545-586(1971).

5. T, J. R. Hughes, R. L. Taylor and W. Kanoknukulchal, 'A simple and efficient finite element for plate bending', Int.j. mumer. methods eng., 11, 1529-1543 (1977).

6. R. H. MacNeal, ‘A simple quadrilateral shell element', Comp. Struct., 8, 175-183 (1978).

7. S. W. Lee and T. H. H. Pian, 'Improvement of plate and shell finite elements by mixed formulations', A.I.A.A. J., 16, 2934 (1978).

8. R. L. Spilker and N. Munir, 'The hybrid stress model for thin plates', Int.j. numer. methods eng., 15, 1239-1260(1980).

9. G. Prathap and G. R. Bhashyam, 'Reduced integration and the shear flexible beam element', Int.j. numer. methods eng., 18, 195-210(1982).

10. G. Prathap, The curved beam/deep arch/finite ring element revisited', Int.j. numer. methods eng., 21, 389-407 (1985).

11. H. Stolarski and T. Belytschko, 'Membrane locking and reduced integration for curved members', J. Appl. Mech., 49, 172-178 (1982).

12. J. E. Walz, R. E. Fuiton, N. J. Cyrus and R. T. Eppink, 'Accuracy of finite element approximations to structural problems', NASA TN-D 5728 (1970).

13. G. Prathap, 'The poor bending response of the four-node plane stress quadrilateral', Int.j. numer. methods eng., 21, 825$835(1985)$.

14. K. H. Murray, 'Comments on the convergence of finite element solutions', A.I.A.A. J., 4, 815-816 (1966).

15. D. G, Ashwell and R. H. Gallagher, Eds., Finite Elementsfor Thin Shells and Curved Members, Wiley, London, 1076.

16. H. R. Meck, 'Anaccurate polynomial displacement function for finitering elements', Comp. Struct., 11, 265-269(1980).

17. G. Prathap and S. Viswanath, 'An optimally integrated four-node quadrilateral plate bending element', Int. j. numer. methods eng., 19, 831-840 (1983).

18. J. L. Batoz, K. J. Bathe and L. W. Ho, 'Study of three node triangular plate bending elements', Int.j. numer. methods eng., $15,1771-1812(1983)$

19. S. Viswanath and G. Prathap, 'A note on locking in a shear flexible triangular plate bending element', Int. j. numer. methods eng. 19, 305-309 (1983).

20. I. Fried, 'Residual energy balancing technique in the generation of plate bending finite elements', Comp. Struct., 4, 771778 (1974).

21. L Fried aad S. K, Yang, 'Triangular, nine degree of freedom C plate bending element of quadratic accuracy', $Q$. Appl. Math, 31, 303-312 (1973).

21 J. Hyman-Garnet, Crouzet-Pascal and A. B. Pifko, 'Aspects of a simple triangular plate bending finite element', Comp. Struct., 12, 783-785 (1980).

23. G. A. Mohr, 'Application of penalty functions to a curved isoparametric axisymmetric thick shell element', Comp. Struct., 15, 685-690 (1982).

24, E L Wilson, R. L. Taylor, W. P. Doherty and T. Ghabussi, 'Incompatible displacement models', in Numerical and Computer methods in Structural mechanics, (Ed. S. P. Fenves et al.), Academic Press, 1973, pp. 43-57.

25. O. C. Zienkiewicz, The Finite Element Method, 3rd edn, McGraw-Hill, London 1971, p. 400.

26. R. D. Cook, Concepts and Applications of Finite Element Analysis, 2nd edn, Wiley, New York 1981, pp. 139-140.

27. J. H. Argyris, M. Haac and H. P. Mlejnek, 'Some considerations on the natural approach', Comp. Meth. Appl. Mech. Eng., 30, 335-346 (1982).

28. T. J. R. Hughes, R. L Taylor and J. F. Levy, 'A finite element method for incompressible flows', Conf. Finite Element Methods in Flow Problems, St. Margaritha, Italy, pp. 1-16 (1976).

29. J. H. Argyris, P. C. Dunne, T. Angelopoulos and B. Bichat, 'Large natural strains and some special difficulties due to non-linearity and incompressibility in finite elements', Comp. Meth. Appl. Mech. Eng., 4, 219-231 (1974). 